\title{
Discordant Biological and Toxicological Species Responses to TLR3 Activation
}

\author{
William M. Mitchell, ${ }^{*}$ Christopher F. Nicodemus, ${ }^{\dagger}$ William A. Carter, ${ }^{\ddagger}$ Joseph C. Horvath ${ }^{\ddagger}$ and David R. Strayer ${ }^{\ddagger}$
}

From the Department of Pathology, Microbiology, and Immunology, * Vanderbilt University School of Medicine, Nashville, Tennessee; AIT Strategies, ${ }^{\dagger}$ Franconia, New Hampshire; and Hemispherx BioPharma, Inc., ${ }^{\ddagger}$ Philadelphia, Pennsylvania

Accepted for publication

December 16, 2013.

Address correspondence to William M. Mitchell, M.D. Ph.D., Department of Pathology, Microbiology, and Immunology, Vanderbilt University School of Medicine, Nashville, TN 37232. E-mail: bill. mitchell@vanderbilt.edu.

\begin{abstract}
Toll-like receptors (TLRs) are highly conserved type 1 membrane proteins that initiate a multiplicity of transient gene transcriptions, resulting in innate and adaptive immune responses. These essential immune responses are triggered by common TLR pattern recognition receptors of microbial products expressed through the cytoplasmic carboxy-terminal Toll/IL-1 domain. Toll/IL-1 adapter protein cascades are induced by an activated Toll/IL- 1 to induce transient transcription responses. All TLRs, with the exception of TLR3, use an MyD88 adapter to Toll/IL-1 to initiate a proinflammatory cascade. TLR3 uses the toll receptor $3 / 4$ induction factor adapter to initiate a different cytosolic adapter cascade with double-stranded RNA agonists. This non-MyD88 pathway induces both NF- $\kappa$ B and type 1 interferon responses. By using a TLR3-restricted double-stranded RNA agonist, rintatolimod, we demonstrate significant unexpected differences in toxic responses between rats and primates. The mechanism of this differential response is consistent with a relative down-regulation of the NF- $\kappa B$ inflammatory cytokine induction pathway in the cynomolgus monkey and humans, but not observed systemically in rat. Our findings suggest evaluation of TLR3 agonists in drug therapy. (Am J Pathol 2014, 184: 1062-1072; http://dx.doi.org/10.1016/j.ajpath.2013.12.006)
\end{abstract}

Animal modeling is a time-tested standard for the development of novel therapeutics. ${ }^{1}$ The potential of such models to adequately translate findings for use in therapeutic development, however, has recently been questioned. A report by Seok et $\mathrm{al}^{2}$ found a poor correlation between human and murine response to inflammatory stimuli, including responses to trauma, burn, and endotoxemia. Discordant results at the level of gene expression array, temporal gene response patterns, and major signaling pathways, in response to these injuries, were demonstrated across species, but well conserved in humans, pointing toward inadequate and nonpredictive nature of available inflammation murine models. This may further explain the difficulty in translating promising pharmacological compounds into successful novel therapies. Despite the heterogeneity of the patients and the causes of life-threatening trauma, the report found highly correlated genomic response profiles across the patient population, but not across species. Burn, trauma, and endotoxemia yielded highly correlated genomic responses within species, but not across species, rodent to human. We have observed a similar dissociation of toxicities in a parallel proinflammatory system, the toll-like receptor 3 (TLR3) induction of innate immune responses.

The TLRs form a family of class 1 transmembrane receptors originally discovered in Drosophila, where they are essential elements in embryogenesis and an evolutionary ancient system of immune response to pathogen-associated molecular patterns (PAMPs). ${ }^{3}$ They were recognized further in plants, ${ }^{4}$ fish, ${ }^{5,6}$ and mice, ${ }^{7}$ as well as primates. ${ }^{7}$ TLRs act as a first line of defense against microbial pathogens by the induction of innate immunity and further provide the initial cellular orchestration for the induction of adaptive immune responses to provide specific humoral and

\footnotetext{
Supported by Hemispherx Biopharma, Inc.

Disclosures: W.M.M. is an independent member of the board of directors and a shareholder of Hemispherx BioPharma, Inc., C.F.N. is a consultant for Hemispherx BioPharma, Inc., W.A.C. is chief executive officer and member of the board of directors and shareholder of Hemispherx BioPharma, Inc., J.C.H. is an employee of Hemispherx BioPharma, Inc., and D.R.S. is the medical director and a shareholder of Hemispherx BioPharma, Inc. Rinitatolimod is an experimental drug whose clinical development and manufacture is funded by Hemispherx BioPharma, Inc., under the trade name Ampligen.
} 
cell-mediated immunity, mediated in part by inflammatory cytokines. ${ }^{8}$ They can be found especially in mature dendritic cells (DCs), central in the host adaptive immune response system. ${ }^{9}$ All of the TLRs use an MyD88-dependent signaling pathway, with the exception of TLR3, which uses the MyD88-independent TRIF pathway. ${ }^{10}$ Two other doublestranded RNA (dsRNA) inducers of gene expression that initiate innate immune responses are the cytosolic helicases, mda5 and retinoic acid inducible gene 1 protein (RIG-1), which are MyD88 dependent. ${ }^{11}$

The PAMP for TLR3 is dsRNA detected in endosomes of antigen-presenting cells and on the cell surface of selected cells, including endothelial cells and airway epithelium. ${ }^{12-14}$ The expression pattern is consistent with sentinel activity for the detection of replicating virus in the host organism. The cellular location of TLR3 is modulated by UNC93B1, which promotes trafficking of differentially glycosylated TLR3 to the plasma membrane; UNC93B1 transcription is up-regulated by dsRNA. ${ }^{15}$

To address the observed toxic heterogeneity of response to PAMPs across species, we have used a restricted TLR3specific agonist, with much data reported in double-blind clinical trials $^{16,17}$ and unpublished open-label safety trials. Rintatolimod (poly I:poly $\mathrm{C}_{12} \mathrm{U}$; Ampligen) is a synthetic dsRNA analogue composed of a single polypurine (inosine) strand and a single polypyrimidine (cytosine and uridine) strand. These are assembled in a dsRNA structure that is maintained under physiological conditions by typical hydrogen bonding between purine and pyrimidine base pairs. The introduction of the pyrimidine base, uridine, at a 1:12 ratio into the polypyrimidine strand maintains the double-stranded structure but allows recurring sites of thermodynamic instability from nonhydrogen bonding of the mismatched base (uridine) with inosine of the polypurine strand. ${ }^{18}$ This small difference in structure, however, restricts induction of transitory gene activities to TLR3, with its unique TRIF signaling pathways, with the exclusion of the mda5 and RIG-1 dsRNA proinflammatory dependence $^{19,20}$ (Figure 1). By using the restricted specificity of rintatolimod and the unique TRIF pathway of TLR3 signaling, we have been able to identify the apparent mechanism for the discordance in TLR3-mediated toxicity between rodents and primates.

\section{Materials and Methods}

\section{Patient Enrollment and Sampling}

Patient serum samples from two clinical trials being conducted according to good clinical practice standards, under
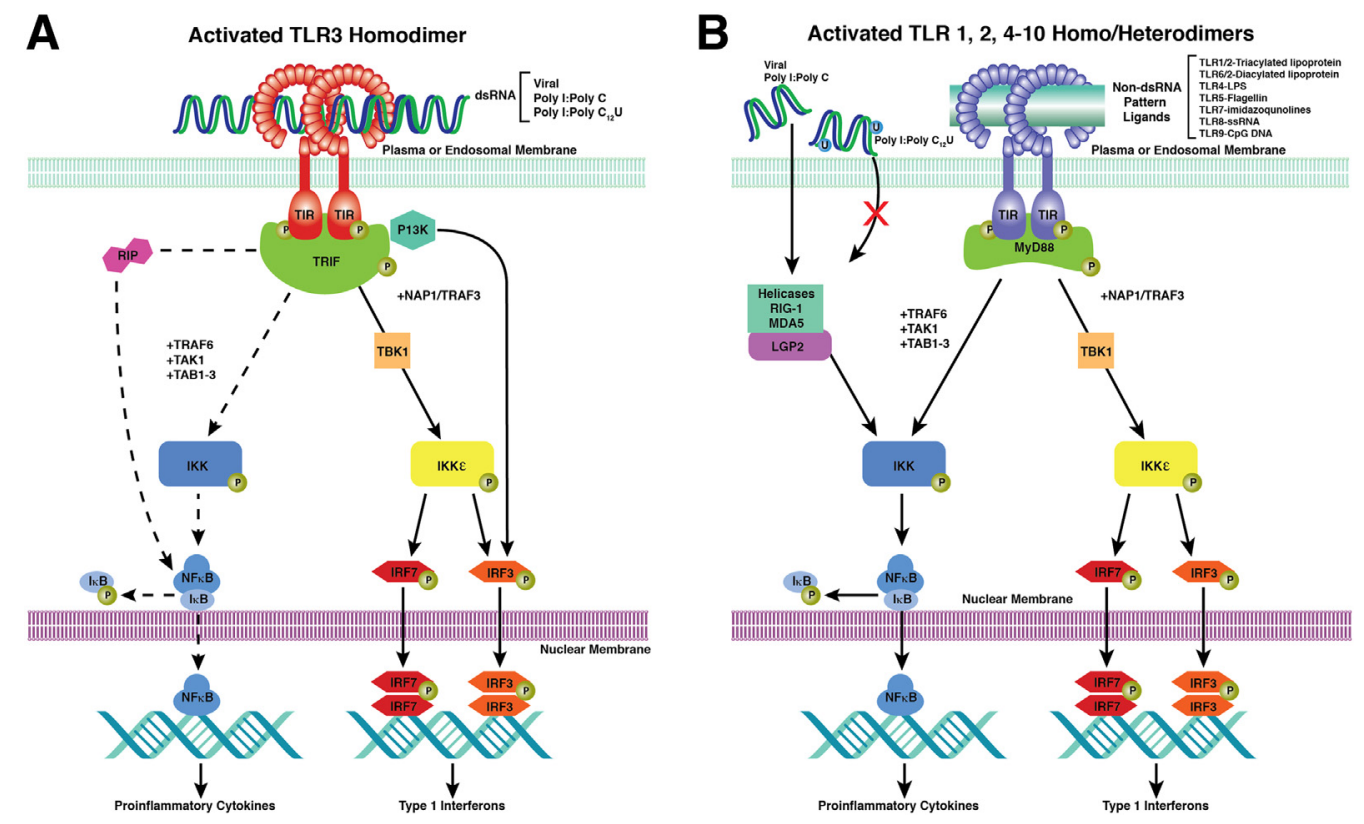

Figure 1 MyD88-independent and MyD88-dependent signaling pathways for the TLRs and helicases. A: The intracellular pathways for MyD88-independent TLR3 nuclear signal transduction initiated by TRIF binding to the TIR of the TLR3 homodimer. TLR3 monomers dimerize with binding of the dsRNA ligand. Activated TRIF initiates two pathways. The first results in the transitory induction of the IFNs. The second is a species-variable pathway (rodents $>>$ primates) that operates through NF- $\mathrm{KB}$ (dashed line) that transiently induces the production of inflammatory cytokines. Adapter protein cascade initiated by TRIF, TBK1, TRAF1/3, NAP1, IKK, IKKE, P13K, IRF-3/7, TAK1, TAB1, and RIP1. The ectodomain of TLR3 consists of a horseshoe-shaped structure populated by 23 leucine-rich $\beta$-sheets (orange disks) connected by nonordered chains containing RNA-binding residues. The transmembrane $\alpha$-helices (solid orange) connect the ectodomain to the cytoplasmic TIR domain (dark green). The phosphorylated TIR binds TRIF to initiate the adapter protein cascade. B: The intracellular pathways for MyD88-dependent signaling for TLR $1 / 2$ and $1 / 6$ heterodimers and TLR 4 to 10 homodimers. The diverse PAMP ligands (green bar) are not necessarily accurate in placement, as is the dsRNA ligand with TLR3 in A. TLR4 uses both the MyD88-dependent and MyD88-independent pathways. TRIF-TIR domain-containing adapter-inducing IFN, TBK1-TANK-binding kinase 1, TRAF-TNF receptor-associated factor, NAP1-Nck-associated protein 1, IKK-IKB kinase, IKK $\varepsilon$ inhibitor of IKB kinase, P13K-phosphoinositide 3-kinase, IRF-IFN regulatory transcription factor, TAB1-TGF- $\beta$-activated kinase 1 , and RIP1 receptor-interacting (TNFRSF) kinase 1. 
the rintatolimod Investigational New Drug No. 39,250, were analyzed for $\gamma$-interferon (IFN), tumor necrosis factor (TNF)- $\alpha$, IL-12p70, and IL-10 levels to assess acute and chronic changes that might be associated with rintatolimod dosing. All human protocols were conducted with Institutional Review Board review and approval and patient informed consent. Study subjects with a diagnosis of chronic fatigue syndrome qualified for participation if they met the protocol entrance criteria. Patients received rintatolimod infusions at $400 \mathrm{mg}$ twice weekly. Frozen serum samples from 14 randomly selected patients in Hemispherx BioPharma's open-label treatment protocol, AMP-511, were obtained at pre-infusion, and again at 4, 24, and 72 hours after infusion. Serum samples in 76 randomly selected patients from the double-blinded protocol, AMP 516, were obtained at baseline pre-infusion and again at week 32 or at early termination if the patient terminated participation before week 32. Serum obtained from clinical testing sites was frozen and shipped to the Hemispherx BioPharma, Inc., testing and manufacturing facility (New Brunswick, NJ), where it was assayed using commercially available enzyme-linked immunosorbent assay kits (human IFN- $\gamma$, human TNF- $\alpha$, human IL-10, and human IL-12p70) (Supplemental Table S1).

\section{Animal Toxicological Analysis}

Animal toxicological analyses were conducted, as required, under investigational new drug guidelines. Studies were conducted according to Good Laboratory Practice procedures at licensed animal pharmacology facilities, according to Institutional Animal Care Committee review and approval. Dose ranging was conducted at Dupont (Wilmington, DE) and BioResearch (Senneville, QC, Canada), and 6-month chronic toxicological studies were conducted at Chrysalis (Cedex, France) and Pharamakon (Waverly, PA). Maximum tolerated doses (MTDs) were derived from acute and subacute dose-ranging studies and were defined as the maximum dose that did not cause mortality or moribund toxicity. Repeat dosing studies were conducted with Sprague-Dawley rats, beagle dogs, cynomolgus monkeys, and New Zealand white rabbits at doses ranging from 0 to $300 \mathrm{mg} / \mathrm{kg}$, i.v. administered daily for 7 to 10 days (rats and rabbits) or twice weekly (monkey and dog) for 4 and 9 weeks, respectively. Clinical observations were recorded during infusions, for 3 hours after infusion, and otherwise twice daily. Complete postmortem analysis was conducted, including gross and microscopic anatomical evaluations using standard animal pathological procedures. Six-month chronic toxicological studies were conducted using twiceweekly doses of $0,6,18$, and $36 \mathrm{mg} / \mathrm{kg}$ rintatolimod under similar procedures.

\section{Animal Cytokine Studies}

Assessment of acute inflammatory cytokines in response to rintatolimod administration was conducted in collaboration with the Lovelace Respiratory Research Institute (Albuquerque, NM) and Hemispherx BioPharma, Inc., under good laboratory practice using an approved animal welfare protocol. Thirty-two Sprague-Dawley rats were infused twice weekly for 8 weeks at $0,6,18$, or $36 \mathrm{mg} / \mathrm{kg}$ and sampled for cytokines at $0,4,24$, and 72 hours after doses 1, 7, and 15, respectively. Each time point after infusion required sacrifice of a male and a female rat. Ten cynomolgus monkeys were dosed for 16 weeks, twice weekly, at the same dosage levels. Serum samples for cytokine measurement were obtained after doses 1, 7, 15, and 30. All serum samples were frozen and assayed for the corresponding patient cytokines using commercially available enzyme-linked immunosorbent assay kits (Supplemental Table S1). All results reported are on the basis of manufacturer-supplied standards yielding linear-dose responses.

\section{Association of Toxicological Findings to the Human Clinical Experience}

Adverse event data in rintatolimod- and placebo-treated patients in controlled studies of chronic fatigue syndrome were assessed in the context of toxicological findings. Adverse events were collected during serial patient evaluations of study subjects participating in prospective 24- to 40week studies of $400 \mathrm{mg}$ rintatolimod or placebo, infused twice weekly for the duration of the protocols. ${ }^{16}$

\section{Molecular Modeling}

The X-ray crystallographic structure of the mouse TLR3 dimer/biol- dsRNA complex and the human TLR3 monomer provided the coordinates for the model of human TLR3, with rintatolimod bound to its active site. Molecular modeling used Accelrys' Discovery Suite software version 2.5.5 (Accelrys, Inc., San Diego, CA). The dsRNA structure of the dimer/biol-dsRNA complex was mutated in situ to the respective poly I (blue) and poly $\mathrm{C}_{12} \mathrm{U}$ (magenta) chains maintaining the phosphate backbone linear translational coordinates of the X-ray crystallographic structure. The coordinates of the human TLR3 monomer were used to replace each homodimer. Several unacceptable close van der Waals contacts for the human TLR3 crystal coordinates were resolved by an alternate rotamer conformation of the individual amino acid $\mathrm{R}$ group. To demonstrate the relative equivalence of these two ligands as TLR3 agonists, we in silico measured the potential energies of the ligands, naked homodimers, and the TLR3-ligand complex. The reduction in potential energy of the complex versus the sum of the potential energy of the components is the energy of binding.

\section{Primary Sequence Comparisons}

Primary protein sequences for the human (GenBank U88879); monkey species, including Macaca mulatta (GenBank BAG55034.1 and AY864735), Macaca fasicularis 
(GenBank BAG55033.1), Papio anubis (XP_003899477), Callithrix jacchus (JAB01765.1), and Saimiri boliviensis (XP_003899477); and rodents, including the house mouse (GenBank AF355152/Mus musculus) and rat (GenBank AB116229/Rattus norvegicus), dog (GenBank XP 005630024/Canis lupus familiaris), and rabbit (GenBank ABB76310/Oryctolagus cuniculus) were aligned using Crystal W software version 10.1.2 provided by DNAStar (Madison, WI).

\section{Results}

\section{Maximum Tolerated Rintatolimod Dose in Multiple Species}

Acute dose-ranging toxicological analysis was completed in the rabbit, dog, rat, and monkey (Table 1). The MTD, defined as the highest acute dosage not associated with induction of a moribund state or mortality, was observed over a range of two orders of magnitude. The rabbit proved most sensitive to rintatolimod, with an MTD of $1.25 \mathrm{mg} / \mathrm{kg}$ per dose. The dog and rat were more tolerant, with MTDs of 10 and $12.5 \mathrm{mg} / \mathrm{kg}$ per dose, respectively. The cynomolgus monkey was substantially more tolerant of rintatolimod, with an MTD of $100 \mathrm{mg} / \mathrm{kg}$ per dose, resulting in a surprisingly 80 -fold differential in acute toxicities, with the nonhuman primate least susceptible to rintatolimod toxicity.

\section{Six-Month Rintatolimod Toxicity in Rats and Monkey}

Because of the clinical development of the rintatolimodenvisioned chronic systemic dosing of the TLR3 agonist over prolonged periods in the treatment of chronic fatigue syndrome and to meet regulatory requirements [Guidance for Industry: Nonclinical Safety Evaluation of Drug or Biologic Combinations; U.S. Department of Health and Human Services, Food and Drug Administration, Center for Drug Evaluation and Research (CDER); http://www.fda.gov/OHRMS/DOCKETS/ 98fr/05d-0004-gdl0002.pdf, last accessed February 3, 2014], 6-month chronic toxicological studies were conducted in the Sprague-Dawley rat and cynomolgus monkey. Animals were dosed similar to the human schedule, with twice-weekly infusions of rintatolimod at 6,18 , or $36 \mathrm{mg} / \mathrm{kg}$ per dose, which were expected to be nonlethal on the basis of the acute toxicity studies. Dose levels were determined by the human dose of 400 $\mathrm{mg}$ per infusion, which is approximately $6 \mathrm{mg} / \mathrm{kg}$ for an average-sized patient. Results of these two chronic toxicological studies were consistent with the findings of the acute toxicological dosing (Table 2), although lethality in the rat during the 6-month course of the study was commonly observed. The rat was extremely sensitive in multiple organs to both the 18- and 36-mg dosing regimens, and many animals in these groups did not survive the 6-month dosing period. In the rat, dose-dependent liver and renal toxicities were observed in all three dosage groups. Additional findings in the rat included anemia and polychromasia, increased alkaline phosphatase,
Table 1 Comparative Species Sensitivity to Rintatolimod MTD

\begin{tabular}{|c|c|c|}
\hline Species & $(\mathrm{mg} / \mathrm{kg})^{*}$ & Dosing in relevant toxicological study \\
\hline Rabbit & 1.25 & $\begin{array}{l}\text { i.v. daily } \times 5 \text { days } \\
(0,1.25,2.5,5 \text {, and } 10 \mathrm{mg} / \mathrm{kg} \text { per dose })\end{array}$ \\
\hline Dog & 10 & i.v. twice weekly $\times \mathrm{mg} / \mathrm{kg}$ per dose \\
\hline Rat & 12.5 & $\begin{array}{l}\text { i.v. twice weekly } \times 13 \text { weeks } \\
(0,12.5,30 \text {, and } 75 \mathrm{mg} / \mathrm{kg} \text { per dose })\end{array}$ \\
\hline $\begin{array}{l}\text { Cynomolgus } \\
\text { monkey }\end{array}$ & 100 & $\begin{array}{l}\text { i.v. twice weekly } \times 4 \text { weeks } \\
(0,12.5,25,50,100 \text {, and } 200 \mathrm{mg} / \mathrm{kg} \\
\quad \text { per dose })\end{array}$ \\
\hline
\end{tabular}

${ }^{*}$ The MTD is defined as the highest dose with no observed mortality or moribund toxicity.

and profound elevation of hepatic transaminases. Histopathological features were consistent with clinical analytes. Dosedependent toxicities were observed in all dose groups, with hepatocellular degeneration, bile duct hyperplasia, and interstitial nephritis with renal tubular dilation. Lymphoid depletion and hyperpigmentation (probably hemosiderin) were noted in the spleen, and extramedullary hematopoiesis and occasional miscellaneous organ inflammation were also noted in all dose groups. In contrast, monkeys tolerated the 6-month course without significant toxicity, except for occasional vomiting associated with infusion in the $36 \mathrm{mg} / \mathrm{kg}$ group. There were transient elevations of hepatic transaminases only in the highdose group, associated with an unexplained decrease in alkaline phosphatase in the monkey. Coagulation parameters were prolonged in the high-dose monkey group. There were no histopathological findings in the monkey, with the exception of increased myelopoiesis in the marrow and follicular thyroid hyperplasia in the 18 and $36 \mathrm{mg} / \mathrm{kg}$ dose groups. The thyroid morphological observation in the nonhuman primate was correlated with an increase in thyroid-stimulating hormone and thyroxine, suggesting a central effect on the pituitary causing thyroid hyperactivity and the observed hyperplasia.

\section{Correlation of Toxicological Patterns in Animals to the Human Clinical Experience}

By using the clinical-grade formulation of poly I:poly $\mathrm{C}_{12} \mathrm{U}$, rintatolimod (Ampligen), we have also found discordance between rodent and human inflammatory responses. As demonstrated with the nonhuman primate model (cynomolgus monkey), humans similarly have minor toxicities compared with the rodent model (Sprague-Dawley rat). As in the models of inflammation explored by Seok et $\mathrm{al}^{2}$ substantial between-species differences are apparent. In contrast to the rodent, in which sensitivity to endotoxin is magnitudes less than in the human, for the rintatolimodmediated TLR3 pathways, the rodent is highly sensitive, whereas primates are relatively tolerant.

The data summarized in Table 3 demonstrate only minimal associations between rodent toxicological findings and the human experience. A low incidence of transient liver 
Table 2 Major 6-Month Chronic Rintatolimod Toxicological Findings in Rat versus Cynomolgus Monkey

\begin{tabular}{|c|c|c|}
\hline Finding & Animal: doses $(\mathrm{mg} / \mathrm{kg})^{*}$ & Description \\
\hline \multicolumn{3}{|l|}{ Clinical } \\
\hline $\begin{array}{l}\text { Pallor, decreased motor activity, } \\
\text { cold to touch, emaciation }\end{array}$ & Rat: 18 and 36 & Increase incidence with dose \\
\hline Abnormal gait & Rat: 18 and 36 & Associated with moribund state \\
\hline Labored breathing & Rat: 18 and 36 & Associated with moribund state \\
\hline Pale fundus & Rat: 18 and 36 & Noted in $25 \%$ of females at 13 weeks \\
\hline Reduced hemoglobin & $\begin{array}{l}\text { Rat: all dose groups } \\
\text { Monkey: only at } 36\end{array}$ & Anemia and polychromasia \\
\hline Increased leukocytes & Monkey (mostly 36) & Increase in CD4 and CD8 T cells throughout the study \\
\hline $\begin{array}{l}\text { Prolonged activated partial } \\
\text { thromboplastin time }\end{array}$ & Monkey: 36 & Prolonged relative to controls, dose response, some effect at $18 \mathrm{mg} / \mathrm{kg}$ \\
\hline Variable alkaline phosphatase & $\begin{array}{l}\text { Monkey: } 18 \text { and } 36 \\
\text { Rat: } 18 \text { and } 36\end{array}$ & $\begin{array}{l}\text { Reduced, especially at highest dose in monkey } \\
\text { Increased in rat }\end{array}$ \\
\hline Increased creatinine & Rat: 36 & Increased in some animals \\
\hline Increased TSH and T4 & Monkey: 18 and 36 & Elevation, dose-response pattern \\
\hline \multicolumn{3}{|l|}{ Pathological features } \\
\hline Liver & Rat: all doses & Varying degrees of hepatocellular degeneration and bile duct hyperplasia \\
\hline Kidney & Rat: all doses & $\begin{array}{l}\text { Minimal to moderate interstitial nephritis and tubular dilatation with } \\
\text { casts }\end{array}$ \\
\hline Spleen & Rat: all doses & $\begin{array}{l}\text { Variable hyperpigmentation; at higher doses, pigmented macrophages in } \\
\text { splenic capsule, lymphoid depletion, and extramedullary hematopoiesis }\end{array}$ \\
\hline Occasional other organ & Rat & $\begin{array}{l}\text { In moribund animals, also pancreatitis, pyelonephritis, prostatitis, and } \\
\text { thymic involution }\end{array}$ \\
\hline Thyroid hyperplasia & Monkey: 18 and 36 & Follicular hyperplasia \\
\hline
\end{tabular}

*Dose-dependent toxicities in 6-month rintatolimod administered i.v. at 0, 6, 18, and $36 \mathrm{mg} / \mathrm{kg}$ doses.

$\mathrm{TSH}$, thyroid-stimulating hormone; $\mathrm{T} 4$, thyroxine.

function test abnormalities is seen in the human (ie, easily manageable, with dose reduction from 400 to $200 \mathrm{mg}$ ). Some patients experience rigors and flu-like symptoms in association with infusion but not the vomiting noted in the high-dose monkey infusion or the general toxicities observed in the rat. No thyroid abnormalities observed in the monkey toxicity trial have been detected in the human clinical data set. Thus, the human experience is similar to the monkey, although a major finding of pituitary-dependent thyroid hyperactivity noted in the monkey was not seen in the human. Both human and monkey differ substantially in pathological response compared with the rodent.

\section{Differences in Expression of Inflammatory Cytokines across Genera}

The unexpected differential toxicities observed between the rat and a nonhuman primate prompted an examination of inflammatory cytokines $(\gamma$-IFN, TNF- $\alpha$, and IL-12p70) associated with infusion of a TLR3 agonist, rintatolimod. Preclinical studies in the rat and cynomolgus monkey assessed the induction of these three inflammatory cytokines, as well as IL-10, measured in the serum in response to three dosage levels $(6,18$, and $36 \mathrm{mg} / \mathrm{kg})$ of rintatolimod administered by i.v. infusion. The 4-hour time point in response to initial infusion in rats gave the strongest systemic signals, which are illustrated in Table 4, with the expression pattern consistent, although less intense, at later time points. The monkey exhibited minimal evidence of systemic cytokine production. A minimal IFN- $\gamma$ response was observed that was significantly different from that in the rat. IL-12p70 at the highest $36 \mathrm{mg} / \mathrm{kg}$ dose level generated a small response in one monkey. In contrast, the rat showed a systemic cytokine response, with signals present for all three inflammatory cytokines and IL-10, even at the lowest $6 \mathrm{mg} / \mathrm{kg}$ dose. The species difference for systemic inflammatory cytokine expression is statistically significant for each cytokine $(P<0.02)$. For comparison with humans, serum was obtained from 14 patients participating in a concurrent open-label clinical study of chronic fatigue syndrome (AMP-511), with a similar time course to the animal pharmacological characteristics and quantified for the same panel of cytokines. The human clinical trial patients were dosed with $400 \mathrm{mg}$ of rintatolimod i.v. over 30 to 60 minutes. Similar to the monkey response, serum cytokine increases were minimally expressed with IFN- $\gamma$, the highest in the aggregate, with a large SD (Table 5). Finally, we assessed for evidence of chronic systemic cytokine changes at the week 32 time point, obtained 
Table 3 Differential Species Toxicities to Rintatolimod

\begin{tabular}{|c|c|c|c|}
\hline \multirow[b]{2}{*}{ Target organ } & \multicolumn{3}{|l|}{ Toxicity } \\
\hline & Rat* $^{*}$ & Monkey* & Human $^{\dagger}$ \\
\hline Liver & $\begin{array}{l}\text { Elevated LFT } \\
\text { Hepatocellular degeneration } \\
\text { Bile duct hyperplasia }\end{array}$ & Elevated LFTs at $36 \mathrm{mg} / \mathrm{kg}$ & $\begin{array}{l}\text { Elevated LFT noted in } 7.4 \% \\
\text { of active and } 1.8 \% \text { of } \\
\text { control patients, managec } \\
\text { with dose reduction }\end{array}$ \\
\hline Kidney & $\begin{array}{l}\text { Increased creatinine, interstitial nephritis, and } \\
\text { renal tubular dilation }\end{array}$ & No findings & No findings \\
\hline Hematopoietic & Anemia all doses, polychromasia & $\begin{array}{l}\text { Anemia at } 36 \mathrm{mg} / \mathrm{kg} \text {, increased } \\
\text { myelopoiesis at all doses }\end{array}$ & One grade 2 anemia $(<2 \%)$ \\
\hline Spleen & Splenic hyperpigmentation and lymphodepletions & No findings & No findings \\
\hline Thyroid & No findings & $\begin{array}{l}\text { Central pituitary stimulation with } \\
\text { thyroid hyperplasia and elevation } \\
\text { of TSH and T4 }\end{array}$ & No thyroid changes \\
\hline
\end{tabular}

*Six-month chronic toxicity studies.

${ }^{\dagger}$ Adverse event data in rintatolimod and placebo-treated patients in well-controlled studies of chronic fatigue syndrome were assessed in the context of toxicological findings. Adverse events were collected during serial patient evaluation of study subjects participating in prospective $24-$ to 40 -week studies of rintatolimod $(400 \mathrm{mg}$ ) or placebo, infused twice weekly for the duration of the study. Elevated LFT results denotes alanine aminotransferase or aspartate aminotransferase greater than three times the upper limit of normal. No patients discontinued secondary to abnormal LFT results.

LFT, liver function test; TSH, thyroid-stimulating hormone; T4, thyroxine.

from 72 patients participating in another rintatolimod chronic fatigue clinical trial (AMP-516). Table 5 illustrates results similar to those of the monkey, with one patient exhibiting a relatively high systemic TNF- $\alpha$ level.

Systemic inflammatory cytokines, observed in rats, are correlated with significant acute and chronic in vivo toxicities at doses that elicited no detectable systemic inflammatory cytokines and minimal toxicities in primates. Moreover, the elevated thyroid activity in the cynomolgus monkey has not been detected in humans. Again, systemic signals of cytokine change in association with 32 weeks of rintatolimod at $400 \mathrm{mg}$ per dose i.v., twice weekly, were not evidenced. Human DCs do show phenotypic changes in association with exposure to rintatolimod, including shift in maturation profile and local release of cytokines (IL-12p70, ILs 4,6 , and 10 , IFN- $\gamma$, and TNF- $\alpha$ ) and the chemokines, monocyte chemoattractant protein-1 (CCL2), and macrophage inflammatory protein-1 $\alpha$ (CCL3) in the microenvironment of culture supernatants. ${ }^{21,22}$ As demonstrated in this communication, systemic elevations of inflammatory cytokines are observed in rats. In monkeys and humans, any local elevations (microenvironment) induced by rintatolimod were rarely detected systemically. The difference is in the magnitude of the response. It is this difference in humans that appears to be responsible, at least in part, with the relative lack of toxicity in primates. Toxicities associated with overwhelming infection are associated with systemic cytokine storms $^{23,24}$ of elevated measurable circulating cytokines. The lack of systemic cytokine detection is consistent with the observed minimal toxicity in primates, in contrast to the significant toxicities observed in nonprimates.

Rat toxicity correlates with systemic cytokine levels, and the lack of primate toxicity similarly is correlated with the lack of significant systemic inflammatory cytokine levels.
In vitro data, however, demonstrate that cells expressing TLR3 respond to dsRNA. ${ }^{21,22}$ The difference between the systemic cytokine levels and the microenvironment is quantitative. Inflammatory cytokines observed in the systemic circulation in rats are associated with extreme toxicity and are analogous to the toxicity seen in humans with lethal viral infections, such as highly pathogenic avian influenza $(\mathrm{H} 5 \mathrm{~N} 1 / \mathrm{H} 7 \mathrm{~N} 9)^{23}$ and severe acute respiratory syndrome (SARS coronavirus) ${ }^{24}$ with inflammatory cytokine storms. The standard human bioactive dose (approximately $6 \mathrm{mg}$ / $\mathrm{kg}$ ) is associated with substantial systemic toxicity in rodents, in which a dose only three times higher is often lethal.

\section{Equivalence of Agonist Binding to TLR3}

Poly I:poly $\mathrm{C}$ is associated with significant human toxicity ${ }^{25}$ not observed with rintatolimod. We examined whether this dissociation of toxicities in humans is related to the affinities of these dsRNA ligands to TLR3. We modeled the predicted relative binding constants of poly I:poly $\mathrm{C}$ versus their mismatched poly $\mathrm{C}$ analogues. Figure 2 demonstrates an in silico molecular model of rintatolimod bound to the human TLR3 ectodomain, forming a homodimer structure, and its close $2 \AA$ amino acid contacts on the TLR3 variable nonhelical segments. These noncovalent contacts provide the collective energetics of binding to form the active homodimer. poly I:poly $\mathrm{C}$ demonstrates equivalent in silico energetics of binding with rintatolimod and several other structures with mismatched bases in the poly $\mathrm{C}$ strand (Table 6). The insertion of a uridine in the poly $\mathrm{C}$ strand of poly I:poly $\mathrm{C}$ at various ratios of $\mathrm{C} / \mathrm{U}$ has no significant effect on the binding affinity with TLR3. Thus, any biological differences observed between rintatolimod and poly I:poly $\mathrm{C}$ cannot be attributed to a differential affinity of TLR3 binding. 
Table 4 Cytokine Responses to Rintatolimod Biweekly Infusions from Baseline in Rats and Monkeys at 4 Hours

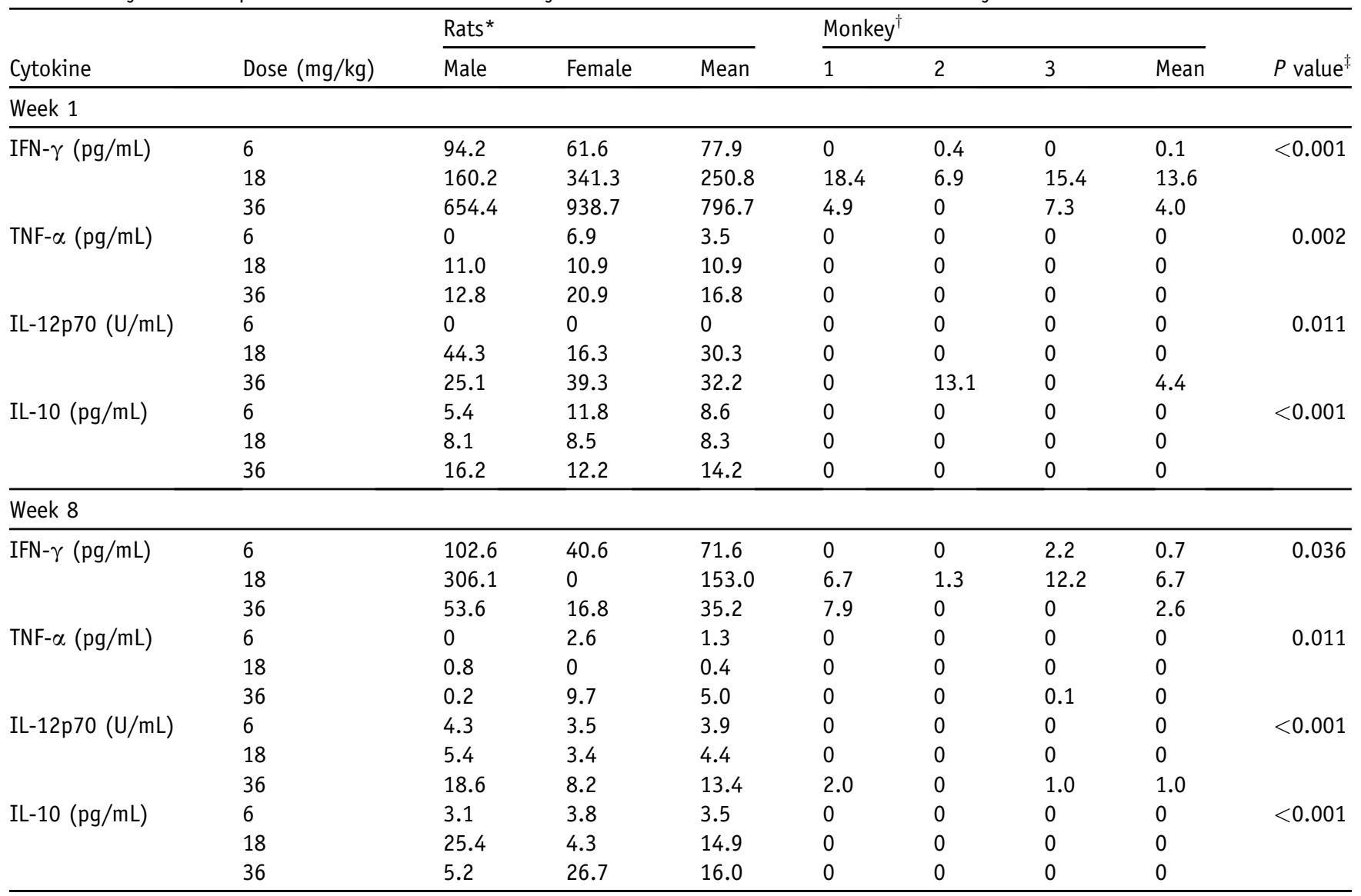

*Each rat (male and female) was sacrificed to obtain blood for analysis of cytokine levels for each cytokine dose for week 1 and week 8 ( $n=12$ ).

${ }^{\dagger}$ Blood was obtained from each monkey by vena puncture for analysis of cytokine levels for each cytokine dose for week 1 and week $8(n=9)$.

${ }^{\ddagger}$ Difference between rat and monkey cytokine levels across the three dosage levels using the Jonckheere-Terpstra test (two sided).

\section{Analysis of Species Sequence Difference}

We evaluated the rabbit (O. cuniculus), dog (C. lupus familiaris), rat ( $R$. norvegicus), and monkey TLR3 coding sequences relative to the human standard for differences in species' orthologs. The rabbit, dog, and rat show 5.4\% sequence nonidentities (Supplemental Figure S1). Monkeys were highly homologous ( $>99 \%$ ) between the five species analyzed (Supplemental Figure S2). The major divergence was as anticipated between old world [M. mulatta, M. fasicularis (cynomolgus), and P. anubis (baboon)] and new world (C. jacchus and S. boliviensis) monkeys. The specific sequence differences between the cynomolgus monkey and rat, relative to humans, are listed in Table 7. Expressed TLR3 protein in the monkey and rat is similar in its total amino acid composition (905 and 906 residues, respectively) versus a

Table 5 Cytokine Responses from Baseline in Patients with Chronic Fatigue Syndrome Enrolled in Two Clinical Trials of Rinitatolimod (Ampligen)

\begin{tabular}{|c|c|c|c|c|c|c|}
\hline \multirow{2}{*}{ Cytokine } & \multirow{2}{*}{\multicolumn{2}{|c|}{$\begin{array}{l}\text { AMP-511 open-label study, } 4 \\
\text { hours after rinitatolimod infusion } \\
\text { Rinitatolimod }(n=14)\end{array}$}} & \multicolumn{4}{|c|}{ AMP-516 placebo-controlled study, week 32} \\
\hline & & & \multicolumn{2}{|c|}{ Rinitatolimod $(n=40)$} & \multicolumn{2}{|c|}{ Placebo $(n=36)$} \\
\hline $\mathrm{IFN}-\gamma(\mathrm{pg} / \mathrm{mL})$ & $3.76 \pm 26.14$ & 0.00 & $1.00 \pm 8.86$ & 0.00 & $11.03 \pm 63.44$ & 0.00 \\
\hline $\mathrm{TNF}-\alpha(\mathrm{pg} / \mathrm{mL})$ & $1.21 \pm 3.30$ & 0.00 & $17.03 \pm 94.73^{*}$ & 1.00 & $1.56 \pm 66.30$ & 0.14 \\
\hline
\end{tabular}

The 400-mg infusions providing an approximately $6 \mathrm{mg} / \mathrm{kg}$ average dosing.

*High means and SD values were secondary to one patient with a value of 500. Removal of this patient results in a mean value of 4.6 . 


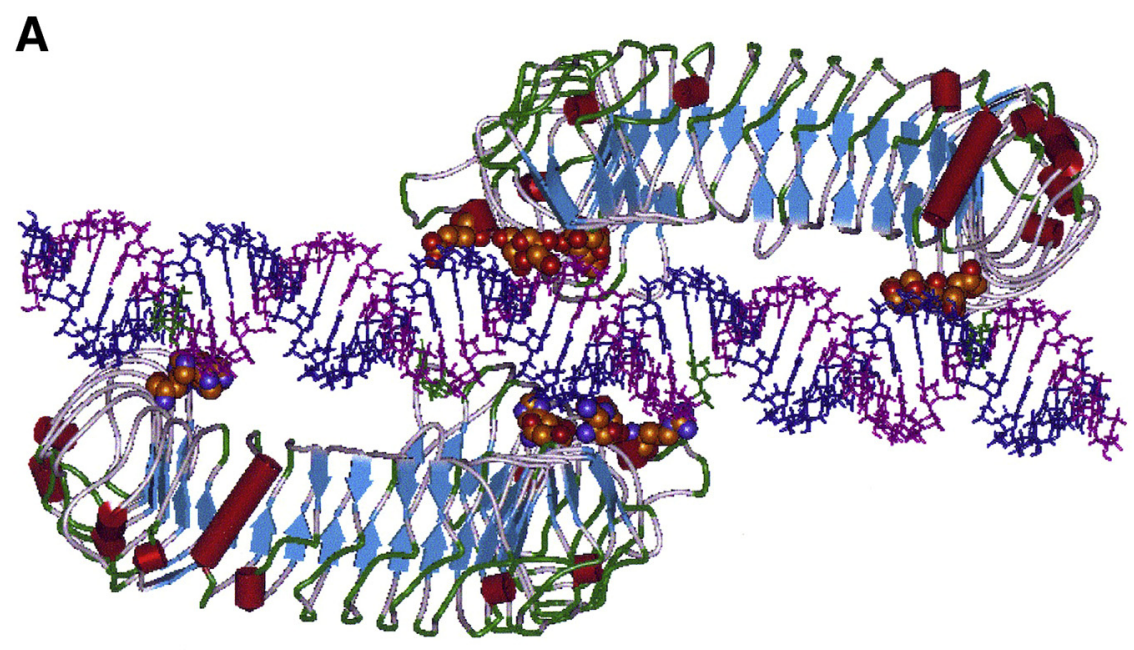

B

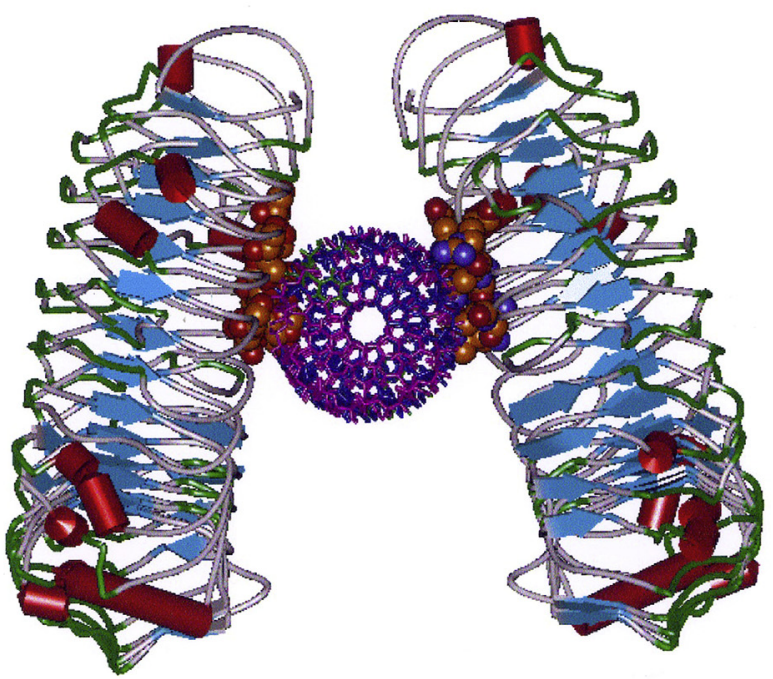

Figure 2 Molecular model of the human TLR3 dimer ecodomain and its rintatolimod ligand. A: View from a lateral perspective of rintatolimod bound to the active site of the TLR3 homodimer. The $\mathrm{C}$-terminal regions of each dimer face each other and bind to the phosphate backbone of the dsRNA. The N-terminals of each TLR3 bind to opposite ends of the dsRNA, with a minimum length of $45 \mathrm{bp}$ required for interaction with essential residues of TLR3 for activation of intracellular signaling. Amino acids of TLR3 required for binding of rintatolimod are shown as CPK (van der Waals' radii) associated with the phosphate backbone. B: The TLR3 homodimer complexed with rintatolimod, as seen down the long axis of the dsRNA. The TLR3 homodimer is represented as structural elements, with the blue arrows signifying direction of the leucine repeat $\beta$ sheets and the red cylinders signifying $\alpha$-helices. poly I strand of rintatolimod (blue) and poly $\mathrm{C}_{12} \mathrm{U}$ strand (magenta cytidines and green uridine). shorter human TLR3 (889 residues). As expected, the monkey TLR3 has greater homology to human (95\%) than mouse/rat sequences $(80 \%$; note that the mouse and rat are $100 \%$ homologous). Rodents have sequence differences versus humans in all of the 23 leucine rich repeats. Differences between humans and monkeys are present in leucine rich repears 2 to 6 and 8 to 22 (20 of 23). The endodomain is more highly conserved between the three species than the ectodomain, probably because of the critical cell signaling function of the cytoplasmic Toll and IL-1 receptor (TIR), which suggests that the monkey and rodent signaling domains may have greater functional similarities than human, on the basis of the large disparity in residue length of human versus monkey and rodent TIR endodomains.

\section{Discussion}

Rintatolimod provided a unique tool that allowed us to dissect the differential effect of species on the systemic response to TLR3 activation. Although poly I:poly $\mathrm{C}$ serves as a ligand for TLR3, and is a prototypic TLR3 agonist for experimental purposes, it also activates the dsRNA-sensing cytosolic helicases. The substitution of a mismatched base (uridine) in the poly $\mathrm{C}$ strand of rintatolimod inactivates its ligand activity for the cytosolic helicases (MDA5 and RIG1) while maintaining its agonist activity for TLR3. ${ }^{19,20}$ The helicases act through the inflammatory MyD88 pathway used by all mammalian TLRs, with the exception of TLR3, which uses the TRIF adapter to initiate innate immune responses. Figure 1 illustrates the pattern recognition of the ectodomain of the TLRs and their cytoplasmic adapter pathways responsible for their pleiotrophic effects. Figure $1 \mathrm{~A}$ depicts the non-MyD88 pathway used by TLR3, and Figure 1B depicts the MyD88-dependent pathways used by all TLRs and the helicases, with the exception of TLR3. Our toxicological analysis demonstrated systemic dose-dependent inflammatory cytokine responses in rats. Although we did not examine inflammatory cytokine responses in rabbit or dog because of a lack of available test kits for the cytokines examined, we believe we would observe a similar quantitative cytokine response in the dog as observed in the rat. The rabbit was so 
Table 6 Interaction Energies of Human TLR3 Complexes with poly I:Cs Containing Variable Mismatched Bases

\begin{tabular}{|c|c|c|c|c|c|c|c|}
\hline Name & $\begin{array}{l}\text { Force } \\
\text { field* }\end{array}$ & $\begin{array}{l}\text { Potential } \\
\text { energy } \\
\text { (kcal/mol) }\end{array}$ & $\begin{array}{l}\text { VDW energy } \\
(\mathrm{kcal} / \mathrm{mol})\end{array}$ & $\begin{array}{l}\text { Electrostatic } \\
\text { energy } \\
(\mathrm{kcal} / \mathrm{mol})\end{array}$ & $\begin{array}{l}\text { VDW interaction } \\
\text { energy }(\mathrm{kcal} / \mathrm{mol})\end{array}$ & $\begin{array}{l}\text { Electrostatic } \\
\text { interaction energy } \\
(\mathrm{kcal} / \mathrm{mol})\end{array}$ & $\begin{array}{l}\text { Net interaction } \\
\text { energy }(\mathrm{kcal} / \mathrm{mol})\end{array}$ \\
\hline huTLR3/poly I: $\mathrm{C}_{12} \mathrm{U}^{\dagger}$ & CHARMM & 1322 & -36 & -1286 & -36 & -1286 & -1322 \\
\hline huTLR3/poly I: $\mathrm{C}_{8} \mathrm{U}$ & CHARMM & 1314 & -36 & -1278 & -36 & -1278 & -1314 \\
\hline huTLR3/poly I: $\mathrm{C}_{16} \mathrm{U}$ & CHARMM & 1301 & -36 & -1265 & -36 & -1265 & -1301 \\
\hline huTLR3/poly I:C & CHARMM & 1323 & -36 & -1287 & -36 & -1287 & -1323 \\
\hline
\end{tabular}

${ }^{*}$ Calculations with the Accelrys Discovery Studio software version 2.5.5 using a distance-dependent dielectric. Net interaction energy is the energy of the complex minus energies of the components.

${ }^{\dagger}$ Poly I: $\mathrm{C}_{12} \mathrm{U}$, rintatolimod.

CHARMM, generalized force field; VDW, van der Waals.

sensitive to rintatolimod that the mechanism may be simply induced by systemic cytokine levels, although other toxicity mechanisms may be involved. There were, however, no significant detectable systemic inflammatory cytokines in either monkeys or humans. The data indicate that the parallel intracellular IFN and NF- $\kappa$ B transcriptional pathways are species dependent in magnitude of response because primate cells in culture respond to rintatolimod, with the biosynthesis of the IFNs and inflammatory cytokines insufficient in vivo to provide a detectable systemic response. The lack of a systemic inflammatory response in primates is consistent with our observation of discordant in vivo toxicities between rats, monkeys, and humans and is consistent with the differences in gross analysis and histopathological characteristics observed between the species. In the case of MyD88-dependent TLR pathways, primates are more sensitive. ${ }^{2}$ In contrast, primates are much more resilient than rodents to inflammatory cytokine toxicity induced by non-MyD88-dependent, TRIF-mediated activation of TLR3. Redundant helicase pathways may reduce the toxicological distinction between species for agonists that activate these pathways, such as poly I:poly $\mathrm{C}$, in contrast to rintatolimod.

Potential mechanisms that may explain the speciesspecific differential toxicities observed with TLR3 activation by dsRNA are apparent on analysis of gene structural differences between species. In addition to the primary sequence differences between rodents and primates illustrated in Table 7, rodent and human TLR3 gene structures are remarkably dissimilar in the proximal promoter domains, as well as TLR3 isoforms. ${ }^{26,27}$ An example of differential functional activity between mice and humans is the induction of TLR3 expression in murine macrophages by lipopolysaccharide (LPS; ie, not seen in humans). ${ }^{7}$ Expression in human blood cells (including monocytes, granulocytes, natural killer cells, T cells, and B cells) and tissue macrophages is undetectable at the transcript level after LPS exposure and is only seen in myeloid DCs. ${ }^{12,28}$ Additional structural differences exist, resulting in possible differential TLR3 isoforms. TLR3 transcripts are initiated in either exon 1 or exon 2 of the mouse, indicating different promoters not present in humans. ${ }^{27}$ The cloning of a human TLR3 isoform ${ }^{29}$ suggests an alternate, but previously unrecognized, splicing pathway. The observed species-specific LPS responsiveness in macrophages could be conferred by consensus motifs for NF- $\kappa \mathrm{B}$ binding present in the murine TLR promoter, which are absent in human promoter. ${ }^{28}$ This has been confirmed by later studies that demonstrate that species-specific differences in tissue expression and responses to LPS coincide with the presence of different evolutionary nonconserved promoter sequences in both species. However, despite the overall nonrelatedness of TLR3 promoter sequences, mRNA expression of both TLR3 orthologs was induced by IFNs, particularly by IFN- $\beta$. The basal and IFN- $\beta$-induced activation of promoters from both species largely depended on similar IFN regulatory factor (IRF) elements, which constitutively

Table 7 Analysis of Monkey and Rodent TLR3 Sequence Orthologs Relative to Human Expressed Protein Sequence

\begin{tabular}{|c|c|c|c|c|c|c|c|c|c|c|}
\hline \multirow[b]{3}{*}{ Domain } & \multicolumn{5}{|l|}{ Monkey } & \multicolumn{5}{|l|}{ Rodent } \\
\hline & \multicolumn{5}{|c|}{ Sequence differences* } & \multicolumn{5}{|c|}{ Sequence differences* } \\
\hline & Total AA & Conservative & Nonconservative & Total & $\begin{array}{l}\% \text { Total } \\
\text { homology }\end{array}$ & Total AA & Conservative & Nonconservative & Total & $\begin{array}{l}\% \text { Total } \\
\text { homology }\end{array}$ \\
\hline Ectodomain & 700 & 11 & 28 & 39 & 94.5 & 700 & 52 & 103 & 155 & 77.9 \\
\hline Endodomain $^{\dagger}$ & $205^{\ddagger}$ & 5 & 1 & 6 & 97.1 & $206^{\S}$ & 14 & 10 & 24 & 88.3 \\
\hline
\end{tabular}

${ }^{*}$ Conservative mutations are those occurring within a similar physical property group: nonpolar/hydrophobic $(A, V, L, P, M, F, W)$, polar uncharged $(G, S, T$, $C, Y, N, Q)$, charged/+ $(K, R, H)$, and charged/- (D, E).

${ }^{\dagger}$ Includes transmembrane and cytoplasmic sequences.

${ }^{\ddagger}$ Total monkey-expressed TLR3 contains 905 residues, versus 889 residues for the human TLR3.

${ }^{\S}$ Total rodent (mouse and rat)-expressed TLR3 contains 906 residues, versus 889 residues for the human TLR3. 
bound IRF-2 and recruited IRF-1 after stimulation. ${ }^{27}$ In murine macrophages, TLR3 up-regulation induced by IFN- $\beta$ required IFNAR1, STAT1, and, in part, IRF-1, but not the Janus kinase family member, Tyk2. Also, LPS specifically up-regulates TLR3 expression through the induction of autocrine/paracrine IFN- $\beta$. In humans, however, IFN- $\beta-$ induced up-regulation of TLR3 was blocked by pretreatment with LPS, despite the efficient induction of IRF- $1 .{ }^{28}$

A recent publication provides additional evidence for differential cytokine responses between mice and humans. ${ }^{30}$ Exposure of human primary DCs to agonist dsRNA did not induce TNF- $\alpha$, IL-6, or IL-8. In human macrophages, the anti-inflammatory cytokine, IL-10, was induced, but TNF- $\alpha$ and IL-6 were not induced. This effect was specific for human cells because TNF- $\alpha$ or IL- 6 was induced by dsRNA in murine DCs. Moreover, there was differential regulation of NF- $\kappa \mathrm{B}$ by murine versus human cells. As shown in numerous prior communications from other laboratories, it was demonstrated that NF- $\kappa \mathrm{B}$ was activated by dsRNA in murine cells but is absent in human DCs and macrophages, as was $\mathrm{I} \kappa \mathrm{B} \alpha$ degradation present in murine cells. ${ }^{30}$

Differences between the ectodomain of humans and nonhuman primates ( 20 of 23 LLRs) provide a potential mechanism to explain the small differential toxicity between the species. Length disparities between the cytoplasmic signaling domain of rodents and nonhuman primates, compared with humans, may contribute to the small differences in toxicity observed between monkeys and humans. There have been numerous reports comparing species specificity of TLR responses, especially TLR3, including mouse/ human, ${ }^{12,27,30,31}$ monkey/human, ${ }^{32}$ and fish/Drosophilal human. ${ }^{5,6}$ A comparison of TLR2 and TLR4 agonists demonstrated similar cytokine levels in humans and chimpanzees versus baboons, consistent with total DNA sequence homologies and the small differences we observed between cynomolgus monkeys and humans. ${ }^{33}$ Although universal TLR responses were largely conserved across primates, chimpanzee-specific immune signaling is enriched for HIVinteracting genes that influence HIV disease progression. ${ }^{34}$ Differences exist in the primate phenotypic responses to TLR agonists. Nevertheless, they are relatively similar when compared with other species.

We have observed significant species differences in the toxic responses to rintatolimod, a restricted TLR3 agonist. Analysis of the dose-dependent toxicities between rats, monkeys, and humans was remarkably dissimilar. Primates are far more resilient to TLR3 stimulation than rodents. The data are consistent with differential TLR3 nuclear transcriptional activities, with the NF- $\kappa \mathrm{B}$ pathway in inflammatory cytokine production in primates playing a relatively minor role compared with the IRF-3/IRF-7 nuclear IFN inducers. Interestingly, rintatolimod has most recently been advanced clinically as a therapeutic modulator of chronic fatigue syndrome, a condition manifested by disordered energy metabolism and symptoms curiously reminiscent of the toxicity associated with inflammatory innate immune responses. It is hypothesized that the therapeutic effect may relate to feedback inhibition in these pathways, with rintatolimod being specific in its activity.

Discordance of toxicity in either direction is equally problematic for the successful clinical development of experimental therapeutics, either placing patients at risk for unexpected toxicity ${ }^{35}$ or, conversely, raising regulatory barriers for agents shown toxic in sensitive species. Alternative methods of in vitro analysis, such as demonstrated by Stebbings et $a l^{36}{ }^{36}$ need to be validated and adapted for regulatory evaluation for human safety. Analogous issues may be encountered in modeling retinal disease across species in the context of RNA-based therapeutics. ${ }^{37}$ The recognition of differential in vivo toxicities and understanding the mechanisms involved should be beneficial in the rational design and risk-benefit analysis of new pharmacological agents for human use.

\section{Acknowledgment}

We thank Angus Shieh (Hemispherx BioPharma, Inc.) for extending his biostatistical expertise in comparative cytokine data analysis.

\section{Supplemental Data}

Supplemental material for this article can be found at http://dx.doi.org/10.1016/j.ajpath.2013.12.006.

\section{References}

1. Woodcock J, Woosley R: The FDA critical path initiative and its influence on new drug development. Annu Rev Med 2008, 59:1-12

2. Seok J, Warren HS, Cuenca AG, Mindrinos MN, Baker HV, Xu W, et al: Genomic responses in mouse models poorly mimic human inflammatory diseases. Proc Natl Acad Sci U S A 2013, 110:3507-3512

3. Rock FL, Hardiman G, Timans JC, Kastelein RA, Bazan JF: A family of human receptors structurally related to Drosophila Toll. Proc Natl Acad Sci U S A 1998, 95:588-593

4. Jebanathirajah JA, Peri S, Pandey A: Toll and interleukin-1 receptor (TIR) domain-containing proteins in plants: a genomic perspective. Trends Plant Sci 2007, 7:388-391

5. Meijer AH, Gabby Krens SF, Medina Rodriguez IA, He S, Bitter W, Ewa Snaar-Jagalska B, Spaink HP: Expression analysis of the Toll-like receptor and TIR domain adaptor families of zebrafish. Mol Immunol 2004, 40:773-783

6. Rodriguez MF, Wiens GD, Purcell MK, Palti Y: Characterization of Toll-like receptor 3 gene in rainbow trout (Oncorhynchus mykiss). Immunogenetics 2005, 57:510-519

7. Alexopoulou L, Holt AC, Medzhitov R, Flavell RA: Recognition of double-stranded RNA and activation of NFkB by Toll-like receptor 3 . Nature 2001, 413:732-738

8. Iwasaki A, Medzhitov R: Toll-like receptor control of the adaptive immune responses. Nat Immunol 2004, 5:987-995

9. Banchereau J, Steinman RM: Dendritic cells and the control of immunity. Nature 1998, 392:245-252

10. Bagchi A, Herrup EA, Warren HS, Trigilio J, Shin HS, Valentine C, Hellman J: MyD88-dependent and MyD88-independent pathways in synergy, priming, and tolerance between TLR agonists. J Immunol 2007, 178:1164-1171 
11. Thompson MR, Kaminski JJ, Kurt-Jones EA, Fitzgerald KA: Pattern recognition receptors and the innate immune response to viral infection. Viruses 2011, 3:920-940

12. Muzio M, Bosisio D, Polentarutti N, D'amico G, Stoppacciaro A, Mancinelli R, van't Veer C, Penton-Rol G, Ruco LP, Allavena P, Mantovani A: Differential expression and regulation of toll-like receptors (TLR) in human leukocytes: selective expression of TLR3 in dendritic cells. J Immunol 2000, 164:5998-6004

13. Kleinman ME: iRNA via TLR3. Nature 2008, 452:591-597

14. Yu M, Levine SJ: Toll-like receptor, RIG-I-like receptors and the NLRP3 inflammasome: key modulators of innate immune responses to doublestranded RNA viruses. Cytokine Growth Factor Rev 2011, 22:63-72

15. Pohar J, Pirher N, Benčina M, Manček-Keber M, Jerala R: The role of UNC93B1 protein in surface localization of TLR3 receptor and in cell priming to nucleic acid agonists. J Biol Chem 2013, 288:442-454

16. Strayer DR, Carter WA, Stouch BC, Stevens SR, Bateman L, Cimoch PJ, Lapp CW, Peterson DL; Chronic Fatigue Syndrome AMP516 Study Group, Mitchell WM: A double-blind, placebo-controlled, randomized, clinical trial of the TLR-3 agonist rintatolimod in severe cases of chronic fatigue syndrome. PLoS One 2012, 7:e31334

17. Strayer DR, Carter WA, Brodsky I, Cheney P, Peterson D, Salvato P, Thompson C, Loveless M, Shapiro DE, Elsasser W, Gillespie DH: A controlled clinical trial in CFS (chronic fatigue syndrome) using a specifically configured RNA drug, poly(I):poly $\left(\mathrm{C}_{12} \mathrm{U}\right)$. Clin Infect Dis 1994, 18:S88-S95

18. Carter WA, Pitha PM, Marshall LW, Tazawa I, Tazawa S, Ts'o PO: Structural requirements of the $\mathrm{rI} \mathrm{n}-\mathrm{rC} \mathrm{n}$ complex for induction of human interferon. J Mol Biol 1972, 70:567-587

19. Gowen BB, Wong MH, Jung KH, Sanders AB, Mitchell WM, Alexopoulou L, Flavell RA, Sidwell RW: TLR3 is essential for the induction of protective immunity against Punta Toro Virus infection by the double-stranded RNA (dsRNA), poly(I:C12U), but not poly(I:C): differential recognition of synthetic dsRNA molecules. J Immunol 2007, 178:5200-5208

20. Trumpfheller C, Caskey M, Nchinda G, Longhi MP, Mizenina O, Huang Y, Schlesinger SJ, Colonna M, Steinman RM: The microbial mimic poly IC induces durable and protective CD4 $+\mathrm{T}$ cell immunity together with a dendritic cell targeted vaccine. Proc Natl Acad Sci U S A 2008, 105:2574-2579

21. Avril T, de Tayrac M, Leberre C, Quillien V: Not all polyriboinosinicpolyribocytidylic acids (Poly I: C) are equivalent for inducing maturation of dendritic cells: implication for alpha-type-1 polarized DCs. J Immunother 2009, 32:353-362

22. Nicodemus CF, Wang L, Lucas J, Varghese B, Berek JS: Toll-like receptor-3 as a target to enhance bioactivity of cancer immunotherapy. Am J Obstet Gynecol 2010, 202:608-610

23. de Jong MD, Simmons CP, Thanh TT, Hien VM, Smith GJ, Chau TN, Hoang DM, Chau NV, Khanh TH, Dong VC, Qui PT, Cam BV, Ha do Q, Guan Y, Peiris JS, Chinh NT, Hien TT, Farrar J: Fatal outcome of human influenza A (H5N1) is associated with high viral load and hypercytokinemia. Nat Med 2006, 12:1203-1207
24. Nagata N, Iwata N, Hasegawa H, Fukushi S, Harashima A, Sato Y, Saijo M, Taguchi F, Morikawa S, Sata T: Mouse-passaged severe acute respiratory syndrome-associated coronavirus leads to lethal pulmonary edema and diffuse alveolar damage in adult but not young mice. Am J Pathol 2008, 172:1625-1637

25. Absher M, Stinebring WR: Toxic properties of a synthetic doublestranded RNA: endotoxin-like properties of poly I. poly C, an interferon stimulator. Nature 1969, 223:715-717

26. Rehli M: Of mice and men: species variations of Toll-like receptor expression. Trends Immunol 2002, 23:375-378

27. Heinz S, Heinz S, Haehnel V, Karaghiosoff M, Schwarzfischer L, Müller M, Krause SW, Rehli M: Species-specific regulation of Tolllike receptor 3 genes in men and mice. J Biol Chem 2003, 278: 21502-21509

28. Visintin A, Mazzoni A, Spitzer JH, Wyllie DH, Dower SK, Segal DM: Regulation of Toll-like receptors in human monocytes and dendritic cells. J Immunol 2001, 166:249-255

29. Yang E, Shin JS, Kim H, Park HW, Kim MH, Kim SJ, Choi IH: Cloning of TLR3 isoform. Yonsei Med J 2004, 45:359-361

30. Lundberg AM, Drexler SK, Monaco C, Williams LM, Sacre SM, Feldmann M, Foxwell BM: Key differences in TLR3/poly I:C signaling and cytokine induction by human primary cells: a phenomenon absent from murine cell systems. Blood 2007, 110:3245-3252

31. Kurt-Jones EA, Sandor F, Ortiz Y, Bowen GN, Counter SL, Wang TC, Finberg RW: Use of murine embryonic fibroblasts to define Toll-like receptor activation and specificity. J Endotoxin Res 2004, 10:419-424

32. Sanghavi SK, Shankarappa R, Reinhart TA: Genetic analysis of Toll/Interleukin-1 Receptor (TIR) domain sequences from rhesus macaque Toll-like receptors (TLRs) 1-10 reveals high homology to human TLR/TIR sequences. Immunogenetics 2004, 56:667-674

33. Brinkworth JF, Pechenkina EA, Silver J, Goyert SM: Innate immune responses to TLR2 and TLR4 agonists differ between baboons, chimpanzees and humans. J Med Primatol 2012, 41:388-393

34. Barreiro LB, Marioni JC, Blekhman R, Stephens M, Gilad Y: Functional comparison of innate immune signaling pathways in primates. PLoS Genet 2010, 6:e1001249

35. Suntharalingham G, Perry MR, Ward S, Brett SJ, Castello-Cortes A, Brunner MD, Panoskaltsis N: Cytokine storm in a phase I trial of the anti-CD28 monoclonal antibody TGN1412. N Engl J Med 2006, 355: $1018-1028$

36. Stebbings R, Findlay L, Edwards C, Eastwood D, Bird C, North D, Mistry Y, Dilger P, Liefooghe E, Cludts I, Fox B, Tarrant G, Robinson J, Meager T, Dolman C, Thorpe SJ, Bristow A, Wadhwa M, Thorpe R, Poole S: "Cytokine storm" in the phase I trial of monoclonal antibody TGN1412: better understanding the causes to improve preclinical testing of immunotherapeutics. J Immunol 2007, 179:3325-3331

37. Kleinman ME, Kaneko $H$, Cho WG, Dridi S, Fowler BJ, Blandford AD, Albuquerque RJ, Hirano Y, Terasaki H, Kondo M, Fujita T, Ambati BK, Tarallo V, Gelfand BD, Bogdanovich S, Baffi JZ, Ambati J: Short-interfering RNAs induce retinal degeneration via TLR3 and IRF3. Mol Ther 2012, 20:101-108 